\title{
Platelet desialylation correlates with efficacy of first-line therapies for immune thrombocytopenia
}

\author{
Lili Tao ${ }^{1,2+}$, Qingshu Zeng ${ }^{3 \dagger}$, June Li ${ }^{4,5,6}$, Miao Xu ${ }^{4,6}$, Jiajia Wang ${ }^{1,2}$, Ying Pan ${ }^{1,2}$, Huiping Wang ${ }^{1,2}$, Qianshan Tao ${ }^{1,2}$, \\ Yang Chen ${ }^{3}$, Jun Peng ${ }^{7}$, Ming Hou ${ }^{7}$, Arend Jan Gerard Jansen ${ }^{8,9}$, Heyu Ni ${ }^{4,5,6,10^{*}}$ and Zhimin Zhai ${ }^{1,2^{*}}$
}

\begin{abstract}
Immune thrombocytopenia (ITP) is a common autoimmune bleeding disorder. Despite considerable investigation, the pathogenesis of ITP remains incompletely understood, and for many patients, effective therapy is still unavailable. Using murine models and in vitro studies of human blood samples, we recently identified a novel Fc-independent platelet clearance pathway, whereby antibody-mediated desialylated platelets can be cleared in the liver via asialoglycoprotein receptors, leading to decreased response to standard first-line therapies targeting Fc-dependent platelet clearance. Here, we evaluated the significance of this finding in 61 ITP patients through correlation of levels of platelet desialylation with the efficacy of first-line therapies. We found that desialylation levels between different responses to treatment groups were statistically significant $(p<0.01)$. Importantly, correlation analysis indicated response to treatment and platelet desialylation were related $(p<0.01)$, whereby non-responders had significantly higher levels of platelet desialylation. Interestingly, we also found secondary ITP and certain non-ITP thrombocytopenias also exhibited significant platelet desialylation compared to healthy controls. These findings designate platelet desialylation as an important biomarker in determining response to standard treatment for ITP. Furthermore, we show for the first time platelet desialylation in other non-ITP thrombocytopenias, which may have important clinical implications and deserve further investigation.
\end{abstract}

Keywords: Platelet, Immune thrombocytopenia, Antibody, Desialylation, Steroid and IVIG therapy

\section{Introduction}

Immune thrombocytopenia (ITP) is a common clinical bleeding disorder characterized by an immune-mediated clearance of autologous platelets, predominantly through autoantibodies targeting platelet surface receptors GPIIbIIIa and/or GPIb-IX and clearance by phagocytic cells in the reticuloendothelial system via Fc $\gamma$-receptors [1-4]. Low platelet counts place ITP patients at risk for severe bleeding including fatal intracranial hemorrhage. Most therapies for ITP including first-line corticosteroids and immunoglobulin G (IVIG), and last resort splenectomy,

\footnotetext{
* Correspondence: nih@smh.ca; zzzm889@163.com

${ }^{\dagger}$ Equal contributors

${ }^{4}$ Toronto Platelet Immunobiology Group, Keenan Research Centre for Biomedical Science and Department of Laboratory Medicine of St. Michael's Hospital, Toronto, Canada

'Department of Hematology, The Second Affiliated Hospital of Anhui Medical University, Hefei 230601, China

Full list of author information is available at the end of the article
}

mainly target the Fc-dependent clearance pathway via blocking/attenuating Fc-Fcy-R interaction or removal of putative site of platelet clearance [4]. However, the pathogenesis and mechanisms of therapies remain poorly understood and around $15-20 \%$ of ITP patients are inexplicably refractory to first-line therapies, and around $10 \%$ are refractory to splenectomy $[5,6]$. In recent years, murine models and large cohort human studies report antibody specificity (i.e., anti-GPIIbIIIa versus anti-GPIb-IX) may play a significant role in dictating response to therapy in ITP [7-9]; whereby presence of anti-GPIb-IX antibodies results in decreased response to corticosteroids and IVIG [7-9]. Most recently, we reported that anti-GPIbo and some anti-GPIIbIIIa antibodies in humans induced platelet desialylation leading to Fc-independent platelet clearance in the liver via hepatic asialoglycoprotein Ashwell-Morell receptors [10], suggesting antibody-mediated desialylation may be one 
of the underlying mechanisms behind resistance to standard ITP therapies $[8,9,11]$.

In the present study, we sought to address whether increased platelet desialylation was correlated with decreased response to treatment in ITP patients (Additional file 1: Supplementary Material). The platelets of randomly and consecutively enrolled 61 patients diagnosed with primary ITP were tested for desialylation prior to the indicated treatments (Table 1). Fluorescein-conjugated lectins Ricinus communis agglutinin I (RCA-1) and Erythrina cristagalli lectin (ECL) were used to detect desialylated galactose and $\beta$-GlcNAc residues via flow cytometry. We found the platelets of ITP patients had significantly higher desialylation as measured by both RCA-1 and ECL binding compared to those of healthy blood donors $(p<0.05)$ (Fig. 1). The 61 ITP patients subsequently underwent standard first-line therapy independent of platelet desialylation and MAIPA assays. After 1 month of treatment, there were 26 complete responders (CR), 21 responders $(\mathrm{R})$, and 14 non-responders (NR) (Table 1). Retrospective data analysis using KruskalWallis rank sum test revealed NR patients had significantly higher platelet desialylation, as compared to the CR and $\mathrm{R}$ groups $(p<0.01)$. Correlation analysis indicated that efficacy and the desialylation level are related (RCA-1 $r=$ $0.395, p<0.01$; ECL $r=0.391, p<0.01$ ). The higher desialylation, the poorer the efficacy of therapy observed.
To test whether the presence of anti-GPIb $\alpha$ antibodies is associated with the platelet desialylation, we detected antibody using MAIPA in the available 33 patient samples collected prior to treatment. We observed a two- to sixfold increased platelet desialylation in patients with anti-GPIb $\alpha$ antibodies $(n=9)$ compared to that in patients with anti-GPIIbIIIa $(n=14)$ or without detectable antibodies $(n=10)$ (Table 1 and Fig. 1B). However, statistical significance was not reached, which is likely due to small sample size. Future larger studies should be useful in determining direct correlation between anti-GPIb $\alpha$ antibody positivity with platelet desialylation.

Interestingly, we also observed significant platelet desialylation in patients with non-ITP thrombocytopenias including connective tissue diseases (CTD), myelodysplastic syndrome (MDS), and aplastic anemia (AA) $(p<0.001)$ but not acute myeloid leukemia (AML) compared to healthy controls (Table 1 and Fig. 1B). Notably, although RCA-1 and ECL measures different types of deglycosylation, we did not observe significant difference between these two assays, suggesting either of them can be used for the potential diagnosis and prognosis.

In summary, our data demonstrates for the first time that the higher level of platelet desialylation is correlated with non-response to the first-line ITP therapies (likely also splenectomies; Additional file 1:

Table 1 Platelet desialylation of different groups $\left[M\left(P_{25}, P_{75}\right)\right]$

\begin{tabular}{|c|c|c|c|c|c|}
\hline & Age & Gender (M/F) & $\mathrm{PLT}\left(\times 10^{9} / \mathrm{L}\right)$ & RCA-1 (\%) & ECL (\%) \\
\hline $\operatorname{ITP}(n=61)$ & $43 \pm 18$ & $18 / 43$ & $16.0 \pm 12.5$ & $1.60(0.50,8.50)$ & $1.30(0.30,5.05)$ \\
\hline \multicolumn{6}{|l|}{ Efficacy grouping ( $n=61)$} \\
\hline$C R(n=26)$ & $36 \pm 16$ & $4 / 22$ & $16.1 \pm 15.3$ & $1.10(0.30,2.05)$ & $0.85(0.28,1.90)$ \\
\hline $\mathrm{R}(n=21)$ & $44 \pm 19$ & $10 / 11$ & $17.2 \pm 10.5$ & $1.80(0.65,5.75)$ & $1.00(0.30,2.05)$ \\
\hline $\operatorname{NR}(n=14)$ & $52 \pm 17$ & $4 / 10$ & $13.9 \pm 9.7$ & $32.95(4.40,62.20)$ & $20.60(2.83,34.68)$ \\
\hline \multicolumn{6}{|l|}{ Antibody grouping ( $n=33)$} \\
\hline Anti-GPIba $(+)(n=9)$ & $39 \pm 14$ & $2 / 7$ & $10.7 \pm 5.3$ & $2.50(0.55,24.15)$ & $2.20(0.45,13.85)$ \\
\hline Single anti-GPIlbllla $(+)(n=14)$ & $35 \pm 16$ & $3 / 11$ & $16.0 \pm 14.5$ & $0.55(0.18,1.70)$ & $0.35(0.10,1.90)$ \\
\hline Double negative $(n=10)$ & $41 \pm 17$ & $5 / 5$ & $14.9 \pm 9.7$ & $0.65(0.10,5.50)$ & $1.15(0.10,2.15)$ \\
\hline CTD $(n=10)$ & $43 \pm 20$ & $3 / 7$ & $20.3 \pm 20.0$ & $5.15(1.63,28.85)$ & $2.20(0.90,14.25)$ \\
\hline $\operatorname{MDS}(n=10)$ & $51 \pm 27$ & $3 / 7$ & $29.3 \pm 18.4$ & $8.75(1.30,14.03)$ & $5.60(2.08,16.85)$ \\
\hline $\mathrm{AA}(n=6)$ & $31 \pm 11$ & $4 / 2$ & $28.2 \pm 9.6$ & $0.75(0.18,18.3)$ & $0.95(0.10,3.05)$ \\
\hline $\operatorname{AML}(n=8)$ & $49 \pm 19$ & $4 / 4$ & $19.4 \pm 18.6$ & $0.20(0.13,0.80)$ & $0.03(0.01,0.50)$ \\
\hline Healthy control $(n=20)$ & $41 \pm 12$ & $10 / 10$ & $197.7 \pm 61.7$ & $0.10(0.10,0.30)$ & $0.00(0.00,0.10)$ \\
\hline
\end{tabular}

The platelets of primary ITP patients were collected prior to treatment. Fluorescin-conjugated lectins RCA-1 and ECL were used to detect desialylated galactose and $\beta$-GIcNAc residues on platelets via flow cytometry. Platelets from healthy blood donors (controls) and secondary ITP and non-ITP thrombocytopenic patients were also studied. Normal distribution measurement data is presented as mean $\pm \mathrm{SEM}$; skewed distribution measurement data is presented as $M\left(P_{25}, P_{75}\right)$, in which $M$ represents the median, $P_{25}$ and $P_{75}$ represent the 25 th percentile and 75th percentile, respectively. Kruskal-Wallis rank sum test showed platelet desialylation is significantly higher in ITP patients as compared to that in healthy blood donors (RCA-1 $Z=-4.918, p<0.001$; ECL $Z=-5.512, p<0.001$ ). The course of therapies was independent from the platelet desialylation assays. The RCA-1 and ECL-positive platelets in non-responder (NR) group are significantly higher than those in complete responder (CR) and responder (R) groups (RCA-1 $\left.X^{2}=10.581, p<0.01 ; E C L X^{2}=13.741, p<0.005\right)$. No significant difference was observed between $C R$ and $R$ groups $(p>0.05)$. Correlation analysis indicated that as platelet desialylation increases, the efficacy of therapy decreases. Higher platelet desialylation in ITP patients with anti-GPlba antibodies was observed as compared with other ITP patients although statistical difference was not reached $\left(\mathrm{RCA}-1 \mathrm{X}^{2}=3.729,0.16>p>0.05\right.$; $\mathrm{ECL} \mathrm{X}^{2}=3.864,0.15>p>0.05$ ). Higher levels of platelet desialylation were also observed in patients of CTD (systemic lupus erythematosus, $n=6$; sicca syndrome, $n=4$ ) with thrombocytopenia; MDS and AA as compared with healthy controls (RCA-1 $X^{2}=33.790, p<0.001 ; E C L X^{2}=42.992, p<0.001$ ). There is no statistical difference in platelet desialylation between the AML patients and healthy donors $(p>0.05)$ 


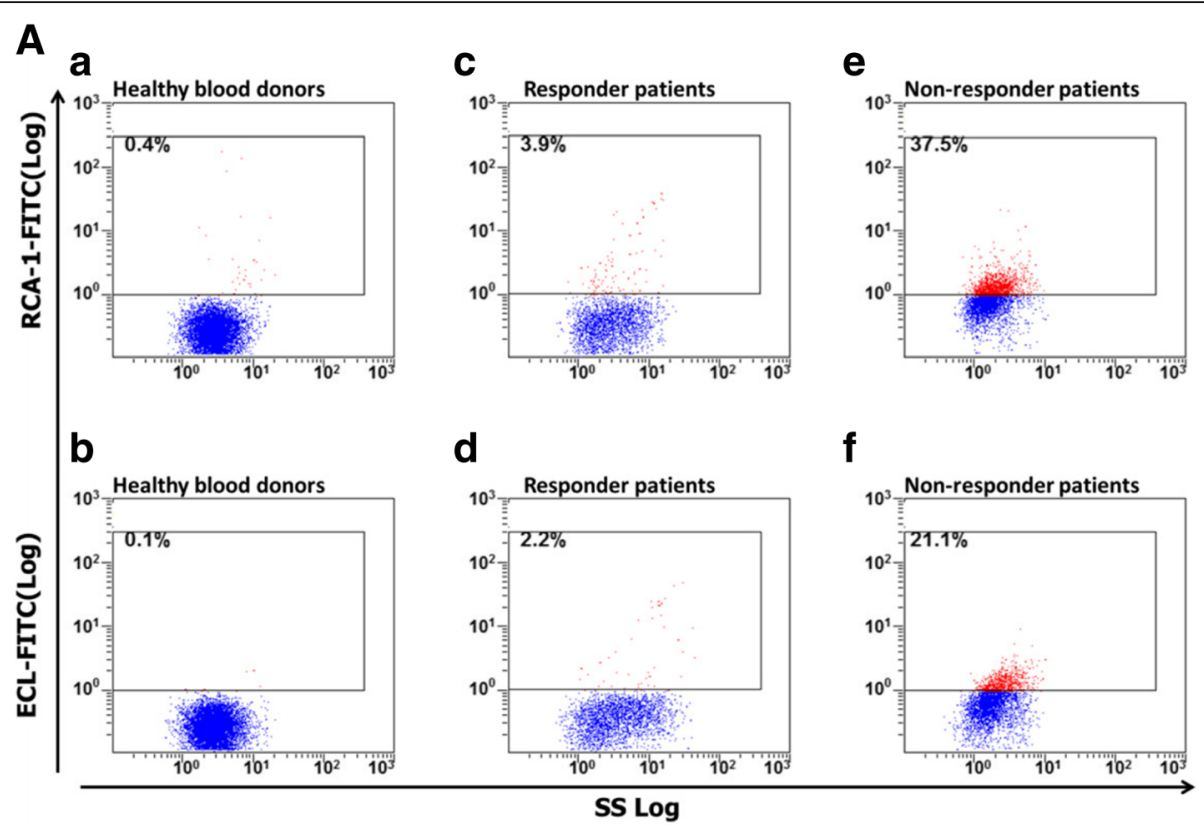

B a

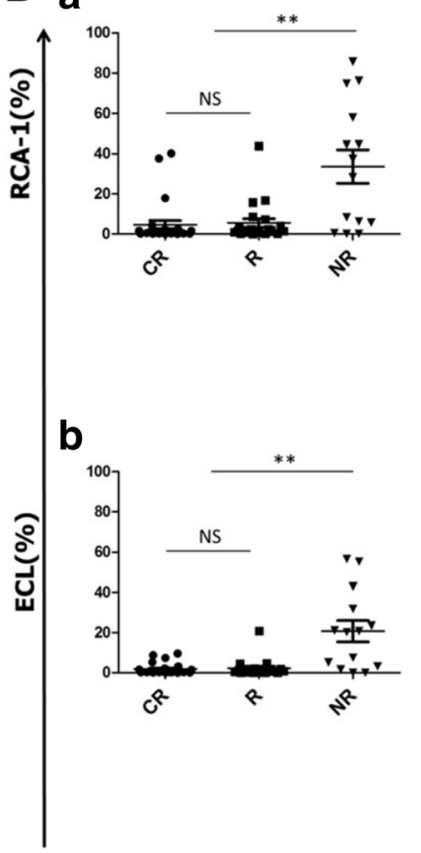

C

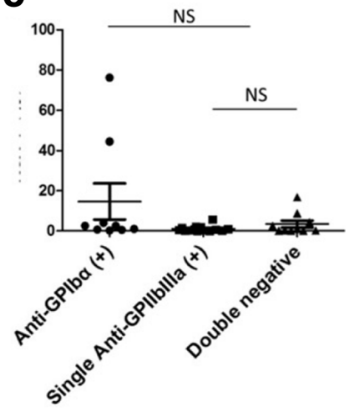

d

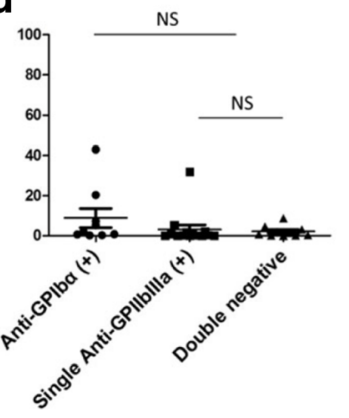

e

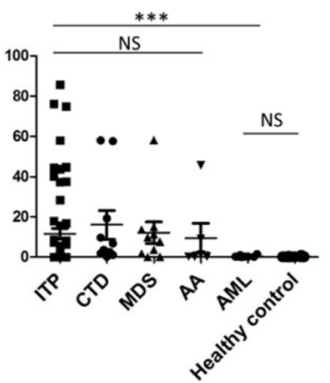

f

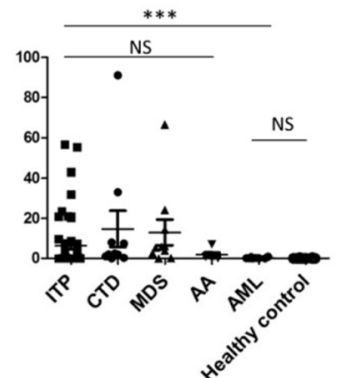

Different groups

Fig. 1 A Platelet desialylation in representative patients and healthy controls. The RCA-1 and ECL binding to platelets from healthy blood donors $(\mathbf{a}, \mathbf{b})$, responder $(\mathbf{c}, \mathbf{d})$, and non-responder patients $(\mathbf{e}, \mathbf{f})$ were examined by flow cytometry. The representative dot plots from each group are shown. B Platelet desialylation in different patient groups and healthy controls. The RCA-1 and ECL levels (Mean \pm SEM) in complete responders $(\mathrm{CR})$, responders (R) and non-responders (NR) $(\mathbf{a}, \mathbf{b})$; anti-GPIba antibody positive (+) group, single anti-GPIIb/llla antibody $(+)$ group, and double negative group (c, d); and in the thrombocytopenias/controls (e, f) (ITP, CTD, MDS, AA, AML, and healthy controls) were examined by flow cytometry. Each point represents the level of platelet desialylation of an individual patient or healthy blood donor $\left({ }^{* *} p<0.01\right.$; $\left.{ }^{* *} p<0.001\right)$

Supplementary Material). These findings not only suggest that platelet desialylation is a useful biomarker in predicting response to treatment in clinical ITP but positions sialidase inhibitors, such as Tamiflu [12], as a potential novel therapeutic in the treatment of ITP as well as other thrombocytopenias. 


\section{Additional file}

Additional file 1: Supplementary Material. (DOCX 49 kb)

\begin{abstract}
Abbreviations
AA: Aplastic anemia; AML: Acute myeloid leukemia; CR: Complete responder; CTD: Connective tissue disease; ECL: Erythrina cristagalli lectin; FITC: Fluorescein isothiocyanate; ITP: Immune thrombocytopenia; IVIG: Intravenous immunoglobulin G; MAIPA: Monoclonal antibody immobilization of platelet antigen assay; MDS: Myelodysplastic syndrome; NR: Non-responder;
\end{abstract} PRP: Platelet-rich plasma; R: Responder; RCA-1: Ricinus communis agglutinin I

\section{Acknowledgements}

The authors would like to thank Mr. Thomas McKeown, Miss Xun Fu, and Miss Jade Sullivan for editing the manuscript.

\section{Funding}

This work was supported by the Canadian Institutes of Health Research (MOP 97918, MOP 119540, MOP 119551); Canadian Blood Services-Canadian Institutes of Health Research partnership fund (CIHR-BUC201403-HN-326400); by the Special Research Funding for the Doctoral Program (NO 20103420110001), Ministry of Education, People's Republic of China; and by the Foundation of the Second Affiliated Hospital and the Hematological Research Center, Anhui Medical University, People's Republic of China. June $\mathrm{Li}$ is a recipient of Ph.D. Graduate Fellowship from Canadian Blood Services. Miao $\mathrm{Xu}$ is a recipient of the State Scholarship Fund from the China Scholarship Council and Ontario Trillium Scholarship, Canada.

\section{Availability of data and materials}

Data sharing is not applicable to this article as no datasets were generated or analysed during the current study.

\section{Authors' contributions}

LT planned and carried out the experiments, analyzed the data, and prepared the manuscript. QZ supervised the clinical studies and analyzed the data. $L$ and MX analyzed the data and prepared the manuscript. JW, YP, HW, QT, and YC carried out the experiments and analyzed the data. AJGJ, JP, and $\mathrm{MH}$ joined the international collaboration group meetings, analyzed the data and contributed to the preparation of manuscript. HN and ZZ (PIs) supervised the research, analyzed the data, and prepared the manuscript. LT and QZ contributed equally to this work and should be acknowledged as co-first authors. Both Dr. HN and Dr. ZZ should be acknowledged as co-corresponding authors. All authors read and approved the final manuscript.

\section{Authors' information}

The contact information for the corresponding authors:

Heyu Ni, M.D.; Ph.D, Professor, Department of Laboratory Medicine and Pathobiology, Department of Medicine, and Department of Physiology, University of Toronto; Scientist of Canadian Blood Services Centre for Innovation; Platform Director for Hematology, Cancer and Immunological Diseases, St. Michael's Hospital, Room 420, LKSKI - Keenan Research Centre, 209 Victoria Street, Toronto, Ontario, M5B 1W8, CANADA. Tel: 1-416-847-1738; Email: nih@smh.ca

Zhimin Zhai, M.D.; PhD. Professor, Director of Department of Hematology, The Second Affiliated Hospital and the Hematological Research Center, Anhui Medical University, Hefei 230601, China. Tel: 86-138-5514-7434; Email: zzzm889@163.com

\section{Competing interests}

The authors declare that they have no competing interests.

\section{Consent for publication}

Not applicable.

\section{Ethics approval and consent to participate}

The study has been approved by the Ethic Board of Anhui Medical University (No. 20131038). Patients in this study all signed informed consents to clinical data use at enrolment.

\section{Author details}

'Department of Hematology, The Second Affiliated Hospital of Anhui Medical University, Hefei 230601, China. ${ }^{2}$ The Hematological Research Center of Anhui Medical University, Hefei 230601, China. ${ }^{3}$ Department of Hematology, The First Affiliated Hospital of Anhui Medical University, Hefei 230022, China. ${ }^{4}$ Toronto Platelet Immunobiology Group, Keenan Research Centre for Biomedical Science and Department of Laboratory Medicine of St. Michael's Hospital, Toronto, Canada. ${ }^{5}$ Canadian Blood Services, Ottawa, Canada. ${ }^{6}$ Department of Laboratory Medicine and Pathobiology, University of Toronto, Toronto, Canada. ${ }^{7}$ Department of Hematology and Shandong Provincial Key Laboratory of Immunohematology, Qilu Hospital, Shandong University, Jinan, China. ${ }^{8}$ Department of Hematology, Erasmus MC, Rotterdam, The Netherlands. ${ }^{9}$ Department of Plasmaproteins Sanquin-AMC Landsteiner Laboratory, Amsterdam, The Netherlands. ${ }^{10}$ Department of Physiology, and Department of Medicine, University of Toronto, Toronto, Canada.

Received: 3 January 2017 Accepted: 1 February 2017

Published online: 08 February 2017

\section{References}

1. Rodeghiero F, Michel M, Gernsheimer T, et al. Standardization of bleeding assessment in immune thrombocytopenia: report from the International Working Group. Blood. 2013;121:2596-606.

2. Cines DB, Bussel JB, Liebman HA, et al. The ITP syndrome: pathogenic and clinical diversity. Blood. 2009;113:6511-21.

3. Lazarus $A H$, Semple JW, Cines DB. Innate and adaptive immunity in immune thrombocytopenia. Semin Hematol. 2013;50 Suppl 1:S68-70.

4. Li J, van der Wal DE, Zhu L, et al. Fc-independent phagocytosis: implications for IVIG and other therapies in immune-mediated thrombocytopenia. Cardiovasc Hematol Disord Drug Targets. 2013;13:50-8.

5. Provan D, Newland A. Fifty years of idiopathic thrombocytopenic purpura (ITP): management of refractory itp in adults. Br J Haematol. 2002;118:933-44.

6. Vianelli N, Galli M, de Vivo A, et al. Efficacy and safety of splenectomy in immune thrombocytopenic purpura: long-term results of 402 cases. Haematologica. 2005;90:72-7.

7. Webster ML, Sayeh E, Crow M, et al. Relative efficacy of intravenous immunoglobulin $\mathrm{G}$ in ameliorating thrombocytopenia induced by antiplatelet GPIIbllla versus GPIbalpha antibodies. Blood. 2006;108:943-6.

8. Zeng $Q$, Zhu $L$, Tao $L$, et al. Relative efficacy of steroid therapy in immune thrombocytopenia mediated by anti-platelet GPIIbllla versus GPIbalpha antibodies. Am J Hematol. 2012;87:206-8.

9. Peng J, Ma SH, Liu J, et al. Association of autoantibody specificity and response to intravenous immunoglobulin $\mathrm{G}$ therapy in immune thrombocytopenia: a multicenter cohort study. J Thromb Haemost. 2014;12:497-504.

10. Li J, van der Wal DE, Zhu G, et al. Desialylation is a mechanism of Fcindependent platelet clearance and a therapeutic target in immune thrombocytopenia. Nat Commun. 2015:6:7737.

11. Li J, Callum JL, Lin Y, et al. Severe platelet desialylation in a patient with glycoprotein Ib/IX antibody-mediated immune thrombocytopenia and fatal pulmonary hemorrhage. Haematologica. 2014;99:e61-63.

12. Shao L, Wu Y, Zhou H, et al. Successful treatment with oseltamivir phosphate in a patient with chronic immune thrombocytopenia positive for anti-GPIb/IX autoantibody. Platelets. 2015;26:495-7.

\section{Submit your next manuscript to BioMed Central and we will help you at every step:}

- We accept pre-submission inquiries

- Our selector tool helps you to find the most relevant journal

- We provide round the clock customer support

- Convenient online submission

- Thorough peer review

- Inclusion in PubMed and all major indexing services

- Maximum visibility for your research

Submit your manuscript at www.biomedcentral.com/submit
Ciomed Central 